\title{
Efficacy and safety of domestic and imported gefitinib in patients with advanced non-small cell lung cancer
}

\author{
Huawei Cheng ${ }^{1 \wedge}$, Hongyue Liu², Qiong Du ${ }^{2}$, Hui Zhang ${ }^{1}$, Xiaodan Zhang ${ }^{1}$, Yangkui Wang', \\ Jingjing Shao ${ }^{1}$, Feng Yang ${ }^{1}$, Bo Zhang ${ }^{1}$, Jing $\mathrm{Shi}^{3}$, Yuguo $\mathrm{Liu}^{3}$, Nan $\mathrm{Wu}^{4}$, Silu $\mathrm{Xu}^{4}$, Qing $\mathrm{Wei}^{4}$, \\ Yancai Sun ${ }^{1}$, Qing Zhai ${ }^{2}$, Bo Yu$^{5}$
}

${ }^{1}$ Department of Pharmacy, The First Affiliated Hospital of USTC, Division of Life Sciences and Medicine, University of Science and Technology of China, Anhui Provincial Cancer Hospital, Hefei, China; ${ }^{2}$ Department of Pharmacy, Fudan University Shanghai Cancer Center, Shanghai, China; ${ }^{3}$ Department of Pharmacy, Shandong Provincial Institute of Cancer Prevention, Shandong Cancer Hospital, Jinan, China; ${ }^{4}$ Department of Pharmacy, Jiangsu Cancer Hospital, Nanjing, China; ${ }^{5}$ Department of Pharmacy, Tong Ren Hospital Shanghai Jiao Tong University School of Medicine, Shanghai, China

Contributions: (I) Conception and design: Y Sun, Q Zhai, B Yu; (II) Administrative support: Y Sun, Q Zhai; (III) Provision of study materials or patients: Y Sun, Y Liu, Q Wei, Q Zhai; (IV) Collection and assembly of data: H Cheng, H Liu; (V) Data analysis and interpretation: H Cheng, H Liu; (VI) Manuscript writing: All authors; (VII) Final approval of manuscript: All authors.

Correspondence to: Yancai Sun. Chief Pharmacist, Anhui Provincial Cancer Hospital, Hefei, China. Email: 13349293359@163.com; Qing Zhai. Chief Pharmacist, Fudan University Shanghai Cancer Center, Shanghai, China. Email: zhaiqing63@126.com; Bo Yu. Chief Pharmacist, Tong Ren Hospital Shanghai Jiao Tong University School of Medicine, Shanghai, China. Email: 786355517@qq.com.

Background Gefitinib is a first-generation epidermal growth factor receptor tyrosine kinase inhibitor (EGFR-TKI). It was approved by the U.S. Food and Drug Administration (FDA) for clinical use in 2003. However, gefitinib has only come to China in recent years. Previous studies have not compared the efficacy and safety of domestic and imported gefitinib. Therefore, we conducted this study.

Methods: This study included 227 patients with advanced non-small cell lung cancer (NSCLC) who received gefitinib treatment in four medical institutions: The First Affiliated Hospital of USTC, Division of life Sciences and Medicine, University of Science and Technology of China, Anhui Provincial Cancer Hospital, Fudan University Shanghai Cancer Center, Shandong Provincial Institute of Cancer Prevention and Jiangsu Cancer Hospital, from January 2017 to July 2018. The patients were divided into a Yiruike group (55 patients treated with domestic gefitinib, Yiruike) and an Iressa group (172 patients treated with imported gefitinib, Iressa). Because gefitinib resistance usually occurs within 8-10 months of gefitinib administration, the patients were followed up for one year to observe their conditions and compare the occurrence of adverse reactions between the two groups. Results: The two groups had no significant difference in baseline data. The median progression-free

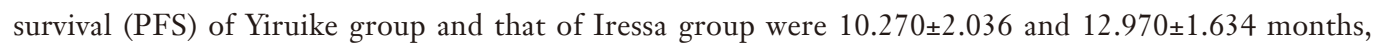
respectively. The mean PFS of Yiruike group and that of Iressa group were 12.598 \pm 1.083 and 15.958 \pm 0.987 months, respectively. The one-year disease control rate (DCR) of Yiruike group and that of Iressa group were $61.8 \%$ and $59.3 \%$, respectively. The differences were all insignificant $(\mathrm{P}>0.05)$. The incidence of adverse reactions in these two groups were not significantly different.

Conclusions: Yiruike was slightly superior to Iressa in terms of DCR. However, comparisons of bioequivalence and DCR were not sufficient for evaluating a drug. Other comparisons require long-term follow-up studies with a large sample size.

Keywords: Gefitinib; non-small cell lung cancer (NSCLC); progression-free survival (PFS); safety

Submitted Jun 12, 2020. Accepted for publication Dec 01, 2020.

doi: 10.21037/apm-20-2140

View this article at: http://dx.doi.org/10.21037/apm-20-2140

\footnotetext{
^ ORCID: 0000-0002-6453-0743.
} 


\section{Introduction}

Lung cancer is among the malignancies with the highest morbidity and mortality in the world and poses a serious threat to human health (1). According to statistics from the National Cancer Center of China, the incidence of lung cancer in China was $0.013 \%$ in 2006-2011, non-small cell lung cancer (NSCLC) cases account for $85 \%$ of all lung cancer cases, $30-40 \%$ of the patients were in the advanced stage at the time of diagnosis, and the overall 5-year survival rate was less than $15 \%(2,3)$. For patients with advanced NSCLC, platinum-based two-drug combination chemotherapy is still the standard first-line treatment, although its response rate is only $30-40 \%$ (4). With the discovery of certain signal transduction pathways in tumor cell growth, new treatment targets have become a research focus. Epidermal growth factor receptor (EGFR) dependent pathways have become important targets in treatment of NSCLC (5). In many solid tumors, EGFR overexpression has been found to be associated with a poor prognosis, a short survival time, and an increased likelihood of tumor metastasis.

Gefitinib inhibits tyrosine kinase activity and affects tumor cell growth by competing with adenosine triphosphate for EGFR binding sites. In 2003, FDA approved gefitinib for the treatment of patients with locally advanced or metastatic NSCLC who have received chemotherapy or have contraindications for chemotherapy (6). Gefitinib (Iressa) was the first successfully developed by AstraZeneca Co., Ltd. Stratified research analysis showed that gefitinib treatment could prolong the duration of adenocarcinoma in oriental, female, non-smoking, and lung median survival time of patients. Iressa came into market in China in 2005, it has shown excellent results in specific population of patients with NSCLC, no matter in the first-line or second-line therapy in the 13-year exploration of targeted therapy for NSCLC in our country. Iressa is expensive, making many patients discouraged, treatment compliance poor. Generic drugs are those produced by other countries and pharmaceutical companies after the original drug has expired. Compared with the original drug, the generic drug has the same active ingredients, dosage form, route of administration and therapeutic effect. Generic drugs can save money, and the reduction of drug cost is an important component of popularization and the rational allocation of public health resources.

Gefitinib (Yiruike), a generic and domestic first-line targeted drug for the treatment of NSCLC, was successfully developed by Qilu Pharmaceutical Co., Ltd. in the 12th Five-Year Plan of China as a major new drug innovation and major science and technology project. Since 2017, Chinese government promoted conformance evaluations of the quality and efficacy of imported and domestic generic drugs. According to the policy, generic drugs which passed the conformance evaluation should be the first choice for clinical use. Yiruike was the only domestic anticancer drug that passed the conformance evaluation by the China Food and Drug Administration in 2017. However, because bioequivalence assessments are the primary method for assessing conformance, the clinical efficacy and safety of Yiruike remains unclear, there has been no large-scale research data on clinical efficacy and adverse reactions in China since this drug was marketed, which is also the design idea of this study.

We present the following article in accordance with the STROBE reporting checklist (available at http://dx.doi. org/10.21037/apm-20-2140).

\section{Methods}

\section{Data source}

This study included 227 patients who were treated with gefitinib for advanced NSCLC between January 2017 and July 2018 in four medical institutions: The First Affiliated Hospital of USTC, Division of life Sciences and Medicine, University of Science and Technology of China, Anhui Provincial Cancer Hospital, Fudan University Shanghai Cancer Center, Shandong Provincial Institute of Cancer Prevention and Jiangsu Cancer Hospital. The study (1.0/2020.5.12) was approved by the Ethics Review Board of the Anhui Provincial Cancer Hospital (review opinion of the Ethics Review Board, Anhui Provincial Cancer Hospital, IRB No. 32, 2020). According to the standards in the Response Evaluation Criteria in Solid Tumors (RECIST, version 1.1), each patient had a measurable lesion on computed tomography (CT), an Eastern Cooperative Oncology Group (ECOG) score no greater than two points, and an estimated survival time of no less than three months. Efficacy was assessed, and all patients were followed up.

\section{Treatment method}

Patients in Iressa group received oral Iressa $(250 \mathrm{mg} / \mathrm{piece}$, 10 pieces/box, unit price: $¥ 2,280$, drug cost: $¥ 228 /$ day) (1 
Table 1 Baseline data of the Iressa and Yiruike groups

\begin{tabular}{|c|c|c|c|}
\hline Baseline data & Yiruike (55 cases) & Iressa (172 cases) & $\mathrm{P}$ \\
\hline Age $\geq 65$ years & $35(63.6 \%)$ & $106(61.6 \%)$ & 0.612 \\
\hline \multicolumn{4}{|l|}{ Smoking } \\
\hline Yes & 6 & 13 & 0.437 \\
\hline \multicolumn{4}{|l|}{ Line of treatment } \\
\hline First line & $38(69.1 \%)$ & $119(69.2 \%)$ & 0.591 \\
\hline Second line & $17(30.9 \%)$ & $45(26.2 \%)$ & \\
\hline Third line & - & $8(4.7 \%)$ & \\
\hline 1 & $36(65.5 \%)$ & $60(34.9 \%)$ & \\
\hline 2 & $17(30.9 \%)$ & $2(1.2 \%)$ & \\
\hline \multicolumn{4}{|c|}{ Recent objective response rate } \\
\hline PR & $5(9.1 \%)$ & $25(14.5 \%)$ & - \\
\hline SD & $16(29.1 \%)$ & $45(26.2 \%)$ & \\
\hline PD & $34(61.8 \%)$ & $102(59.3 \%)$ & \\
\hline
\end{tabular}

ECOG, Eastern Cooperative Oncology Group; PR, partial response; SD, stable disease; PD, progressive disease.

tablet) daily, which can be taken with or without food until disease progression or intolerable side effects occur. Patients in Yiruike group received oral Yiruike $(250 \mathrm{mg} /$ piece, 10 pieces/box, unit price: $¥ 498$, drug cost: $¥ 49.8 /$ day) (1 tablet) daily, which can be taken with or without food until disease progression or intolerable side effects occur. All procedures performed in this study involving human participants were in accordance with the Declaration of Helsinki (as revised in 2013). Informed consent was taken from all the patients.

\section{Evaluation of response rates and adverse reactions}

PFS and DCRs were recorded for the two groups of patients. According to the Common Terminology Criteria for Adverse Events (CTCAE4.03) developed by the U.S. National Cancer Institute, the adverse reactions of all patients were evaluated.

\section{Statistical methods}

The SPSS 23.0 software package was used for data processing. Measurement data were expressed as $\bar{x} \pm \mathrm{s}$ and subjected to an independent-samples $t$-test, and count data were subjected to a chi-square test. The Kaplan-Meier method and log-rank test were used for analyzing the input data, and $95 \%$ confidence intervals were calculated. $\mathrm{P}<0.05$ was considered statistically significant.

\section{Results}

\section{Baseline data}

A total of 227 patients were included in this study and divided into Yiruike group (55 patients treated with domestic gefitinib, Yiruike) and Iressa group (172 patients treated with imported gefitinib, Iressa). Patients with incomplete clinical information collection have been excluded. The baseline data for each group were shown in Table 1.

\section{PFS of the Iressa and Yiruike groups}

The median PFS of Yiruike group and Iressa group was 
$10.270 \pm 2.036$ and $12.970 \pm 1.634$ months, respectively. The mean PFS of the Yiruike group and the Iressa group was $12.598 \pm 1.083$ and $15.958 \pm 0.987$ months, respectively. The chi-square value of the log-rank (Mantel-Cox) test was 0.794 , the $\mathrm{P}$ value was $0.373(\mathrm{P}>0.05)$, and the difference was not statistically significant (Figure 1).

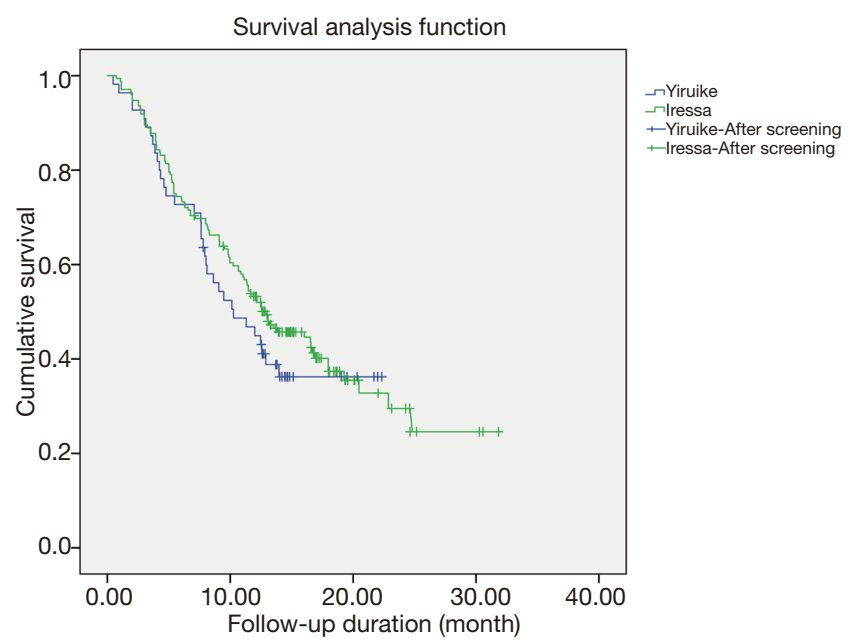

Figure 1 Kaplan-Meier progression-free survival curves for patients treated with Yiruike and Iressa.

\section{One-year DCRs of the Yiruike group and Iressa group}

The patients in the two groups were followed up for one year. In the Yiruike group, 16 patients had a stable condition, and 34 patients had disease progression. In the Iressa group, 45 patients had a stable condition, and $102 \mathrm{had}$ disease progression. The two groups were not significantly different in terms of DCR $(\mathrm{P}>0.05)$. The DCRs of the Yiruike and Iressa groups were listed in Table 2.

\section{Adverse events in the Yiruike and Iressa groups}

Common adverse reactions to gefitinib include rash and diarrhea. Although interstitial pneumonia is a rare adverse reaction to gefitinib, it is life-threatening.

In our study, the two groups of patients had different degrees of adverse reactions, mainly manifested as liver injury, rash, bone marrow suppression, diarrhea, interstitial pneumonia and hand-foot syndrome (Table 1). There was no significant difference in the incidence of adverse reactions between the two groups $(\mathrm{P}>0.05)$ (Table 3).

\section{Discussion}

Gefitinib is one of the first EGFR-TKIs for the treatment

Table 2 DCRs of the Yiruike and Iressa groups

\begin{tabular}{lccc}
\hline & Yiruike $(55$ cases $)$ & Iressa (172 cases) & $P$ \\
\hline DCR & $61.8 \%$ & $59.3 \%$ & $>0.05$ \\
\hline
\end{tabular}

$\mathrm{DCR}$, disease control rate.

Table 3 Adverse reactions in the Yiruike and Iressa groups

\begin{tabular}{lccc}
\hline Adverse reactions & Yiruike $(55$ cases $)$ & Iressa (172 cases) & P \\
\hline Liver injury & 13 & 22 & 0.086 \\
Rash & 10 & 21 & 0.368 \\
Diarrhea & 0 & 3 & 0.573 \\
Hand-foot syndrome & 1 & 2 & 1.000 \\
Stomatitis & 0 & 0 & 0.246 \\
Thrombocytopenia & 1 & 1 & 0.432 \\
Leukopenia & 1 & 0 & 0.246 \\
Neutropenia & 1 & 2 & 1.000 \\
Pruritus & 0 & 2 & 1.000 \\
Pneumonia & 1 & 3 & \\
\hline
\end{tabular}


of locally advanced or metastatic NSCLC in patients with EGFR mutations (7). Compared with chemotherapy, gefitinib can prolong PFS for 8-10 months (8), thus it has become a commonly used targeted drug for advanced NSCLC. Our study evaluated the clinical efficacy and safety of domestic and imported gefitinib in patients with advanced NSCLC, our study may provide effective evidence of the clinical effectiveness of anticancer drugs.

As what mentioned before, gefitinib resistance usually occurs within $8-10$ months of gefitinib administration and due to the limitation of patients life expectancy, we followed the patients for one year to observe their conditions and compare the efficacy and safety between the two groups. We can see all data from the results was not significantly different. These results suggest that the original and genetic drug on the short-term treatment of patients with advanced NSCLC with positive EGFR mutation is relatively consistence. Our study affirms the capability and level of China's pharmaceutical industry from one aspect. There was no statistically significant difference in PFS and DCR between the two groups in our study $(\mathrm{P}>0.05)$, which indicated that although the two groups of patients have taken different drugs, but in the survival situation the benefits were consistent. Although the rate of no disease progression and no death was not high in both groups after 12 months of observation, this was an acceptable prognosis for patients with advanced NSCLC. However, as could be seen from the comparison of treatment costs between the two groups, the monthly costs of patients in Yiruike group were significantly lower than those in Iressa group. The biggest advantage of generics is that they are cheap. Using generic gefitinib can save patients more than $¥ 5,000$ /month with the same benefit, which may be a great significance to the continuity of treatment plan and the compliance of patients, etc. China has a large population base, too many cancer patients, and the level of economic development lags behind. After putting the generic drugs with ideal curative effects into use, it is of positive significance not only for the patients themselves, but also for their relatives, families and even the whole society.

However, drugs are evaluated not just to determine equivalence and disease control. More and longer-term follow-up evaluations still await further studies. This study is our newest attempt, and we will continue to collaborate with other cancer centers to conduct more real-world studies to fill gaps in drug evaluation.

\section{Acknowledgments}

Funding: None.

\section{Footnote}

Reporting Checklist: The authors have completed the STROBE reporting checklist. Available at http://dx.doi. org/10.21037/apm-20-2140

Data Sharing Statement: Available at http://dx.doi. org/10.21037/apm-20-2140

Conflicts of Interest: All authors have completed the ICMJE uniform disclosure form (available at http://dx.doi. org/10.21037/apm-20-2140). The authors have no conflicts of interest to declare.

Ethical Statement: The authors are accountable for all aspects of the work in ensuring that questions related to the accuracy or integrity of any part of the work are appropriately investigated and resolved. The study (1.0/2020.5.12) was approved by the Ethics Review Board of the Anhui Provincial Cancer Hospital (review opinion of the Ethics Review Board, Anhui Provincial Cancer Hospital, IRB No. 32, 2020). All procedures performed in this study involving human participants were in accordance with the Declaration of Helsinki (as revised in 2013). Informed consent was taken from all the patients.

Open Access Statement: This is an Open Access article distributed in accordance with the Creative Commons Attribution-NonCommercial-NoDerivs 4.0 International License (CC BY-NC-ND 4.0), which permits the noncommercial replication and distribution of the article with the strict proviso that no changes or edits are made and the original work is properly cited (including links to both the formal publication through the relevant DOI and the license). See: https://creativecommons.org/licenses/by-nc-nd/4.0/.

\section{References}

1. Siegel RL, Miller KD, Jemal A. Cancer Statistics, 2017. CA Cancer J Clin 2017;67:7-30.

2. Zheng $\mathrm{R}$, Zeng $\mathrm{H}$, Zhang $\mathrm{S}$, et al. National estimates of cancer prevalence in China, 2011. Cancer Lett 2016;370:33-8. 
3. Sathiakumar N, Delzell E, Morrisey MA, et al. Mortality following bone metastasis and skeletal-related events among patients 65 years and above with lung cancer: A population-based analysis of U.S. Medicare beneficiaries, 1999-2006. Lung India 2013;30:20-6.

4. Cimino GD, Pan CX, Henderson PT. Personalized medicine for targeted and platinum-based chemotherapy of lung and bladder cancer. Bioanalysis 2013;5:369-91.

5. Hu X, Chen W, Li X, et al. Clinical efficacy analysis of Osimertinib treatment for a patient with leptomeningeal metastasis of EGFR+ non-small cell lung cancer without the T790M mutation. Ann Palliat Med 2019;8:525-31.

Cite this article as: Cheng $\mathrm{H}$, Liu $\mathrm{H}$, Du Q, Zhang $\mathrm{H}$, Zhang X, Wang Y, Shao J, Yang F, Zhang B, Shi J, Liu Y, Wu N, Xu S, Wei Q, Sun Y, Zhai Q, Yu B. Efficacy and safety of domestic and imported gefitinib in patients with advanced non-small cell lung cancer. Ann Palliat Med 2021;10(1):10-15. doi: 10.21037/ apm-20-2140
6. Rahman AF, Korashy HM, Kassem MG. Gefitinib. Profiles Drug Subst Excip Relat Methodol 2014;39:239-64.

7. Wu YL, Saijo N, Thongprasert S, et al. Efficacy according to blind independent central review: Post-hoc analyses from the phase III, randomized, multicenter, IPASS study of first-line gefitinib versus carboplatin/paclitaxel in Asian patients with EGFR mutation-positive advanced NSCLC. Lung Cancer 2017;104:119-25.

8. Mok TS, Wu YL, Thongprasert S, et al. Gefitinib or carboplatin-paclitaxel in pulmonary adenocarcinoma. $\mathrm{N}$ Engl J Med 2009;361:947-57. 